\title{
Stellar Masses from Double Star Observations with the Mark III Interferometer
}

\author{
J. T. ARMSTRONG \\ NRL/USNO Optical Interferometer Project and USRA
}

\begin{abstract}
By improving on the angular resolution possible with conventional telescopes, speckle interferometry has been adding to the list of known masses, resolving binaries with separations of 30 mas. Interferometry with separate apertures offers the possibility of going to much higher resolution.

The Mark III Optical Interferometer on Mt. Wilson has been used for astrometry, for stellar diameter measurements, and for binary observations since routine operations began in late 1988 . The fringe visibilty calibration uncertainty is $\sim 1 \%$ for $m<4^{\mathrm{m}}$ at $\lambda 800 \mathrm{~nm}$ with good seeing, so that both binary components can be detected when the magnitude difference is $3.5^{\mathrm{m}}$ to $4^{\mathrm{m}}$.

From Mark III data, masses of eight stars have been determined. The most precise determination is for the components of the $\phi$ Cygni system: $2.545 \pm 0.085$ and 2.445 $\pm 0.081 \mathcal{M}_{\odot}$. To improve on this measurement, more precise spectroscopic data are needed, as is the case for most of the systems for which we have orbits or preliminary orbits.

The NRL/USNO Optical Interferometer Project is currently designing two more capable instruments: a four-element system for astrometric observations, and a sixelement system for imaging. The ultimate $430-\mathrm{m}$ maximum baseline and $35-\mathrm{cm}$ apertures of the imaging interferometer will allow observations to $8^{\text {th }}$ to $10^{\text {th }}$ mag and resolution of binary components separated by as little as $\approx 200 \mu$ as.
\end{abstract}

\section{INTRODUCTION}

The lack of overlap between visual and spectroscopic observations of binaries has been cited several times during this conference. The reasons for this lack are obvious: the components of visual binaries are too widely separated to conveniently yield radial-velocity curves, while spectroscopic binaries with observable velocity curves are too close to resolve visually. As a result, the most precise stellar mass measurements come from observations of eclipsing sytems (see $\mathrm{An}$ dersen 1991). In the last ten to twenty years, however, the number of stars with both visual and spectroscopic orbits has increased, owing to the advent of speckle interferometry. Binary components separated by as little as $\mathbf{3 0}$ mas can be resolved by this technique, and a number of mass determinations from double-lined spectroscopic/visual systems has resulted.

Interferometry with separated apertures is now extending the overlap region by resolving binaries with separations of only a few milliarcseconds. At the Naval Research Laboratory, the interferometry group and collaborators have been observing spectroscopic binaries since mid-1988 with the Mark III Optical Interferometer on Mt. Wilson with the aims of measuring their visual orbits and of determining the masses of the components of double-lined systems. In this talk, I will describe the Mark III's capabilities and limitations, show the kind of data and the precision that can be obtained with the Mark III, summarize the results of our mass determinations to date, discuss in more detail two systems in which we have measured the stellar masses, and give a brief description of the 
interferometers that the NRL/USNO interferometry group will soon be installing at the Lowell Observatory.

\section{THE MARK III OPTICAL INTERFEROMETER}

The Mark III Optical Interferometer is a two element fringe-tracking Michelson interferometer sitting in the shadow of the 100-inch Hooker telescope on Mt. Wilson. Mt. Wilson is also the site of Michelson's stellar interferometer, used by Anderson (1920) to measure the separation of the components of Capella. The Mark III is a joint NRL/USNO/JPL/CfA/MIT project, and has been operated continuously since mid- 1988 by NRL. The people involved in the project include Ken Johnston, Richard Simon, Dave Mozurkewich, Andreas Quirrenbach, Christian Hummel, David Buscher, Craig Denison, and myself at NRL; Don Hutter, Nicholas Elias, and the late Jim Hughes at USNO; Mike Shao, Mark Colavita, and Brad Hines at JPL; and Xiao-Pei Pan at Caltech. A brief description of the Mark III is given here. More detail on the instrument and the data reduction are given by Shao et al. (1988), Mozurkewich et al. (1991), and Armstrong et al. $(1992 \mathrm{a})$.

Three programs occupy most of the observing time on the Mark III: astrometry, stellar diameter measurements, and visual orbits of spectroscopic binaries. A typical binary observing list consists of a half dozen program stars and an equal number of unresolved single stars used as fringe visibility calibrators. Scheduling of observations, image acquisition and tracking, and fringe acquisition and tracking are all automatic, permitting us during the best nights to make over 200 scans of 75 seconds of integration each.

We have five siderostats available as interferometer elements, of which two at a time can be used. The three fixed siderostats, used for astrometry, give us a $12-\mathrm{m}$ north-south baseline or a $12-\mathrm{m}$ northeast-southwest baseline. The two moveable siderostats can be placed on any of a set of piers along a northsouth line to give baselines ranging from 3 to 31 meters, corresponding roughly to resolutions of 30 to 3 mas. The siderostats are $25 \mathrm{~cm}$ in diameter, but the apertures of the system are limited to $7.5 \mathrm{~cm}$ in the laboratory, of which the inner $5 \mathrm{~cm}$ is used for fringe detection and tracking. Light is brought from the siderostats to the optics lab in evacuated pipes to eliminate ground seeing effects.

In the lab are two delay lines, each consisting of a retroreflector in a 5-m long evacuated tank, for a total range of $20 \mathrm{~m}$ of adjustable optical path length difference. Active optics are used both to find and track interference fringes and to keep the two stellar images coincident. Our fringe detection technique uses a one-wavelength $250 \mathrm{~Hz}$ path-length modulation to scan over the interference fringes with synchronous detection of the combined light. The difference between the expected and observed fringe positions is used to track the atmospherically induced fringe motions.

On each side of the beam combiner, the combined light is split by a dichroic mirror, giving us four output channels. Channel 1 , which is used only for detecting fringes, is a broadband red $(\approx 700$ to $900 \mathrm{~nm})$ channel. Channels 2 to 4 pass through narrow-band filters, usually $25 \mathrm{~nm}$ wide and usually centered at $\lambda \lambda 800$, 550 , and $500 \mathrm{~nm}$. Light from these three channels, detected synchronously with 
the path-length modulation, gives us the fringe visibility data we use in later data processing. These data are normalized by the visibilities of the calibration. The precision of the calibrated visibilities is limited by photon noise and by systematic effects such as seeing variations with time and air mass.

The capabilities of the Mark III resulting from the aperture sizes, the baseline and delay line lengths, and calibration uncertainties are given in Table 1.

TABLE 1. Mark III Capabilities for Binary Observations

\begin{tabular}{|l|c|l|}
\hline \multicolumn{1}{|c|}{ Quantity } & Limit & \multicolumn{1}{c|}{ Comments } \\
\hline Magnitude limit & $m \$^{5}$ & $\begin{array}{l}\text { 2-inch equivalent apertures for fringe } \\
\text { and image acquisition }\end{array}$ \\
Declination & $\delta>-10^{\circ}$ & $\begin{array}{l}\text { Need senith angle } 550^{\circ} ; \text { limited } \\
\text { declination coverage on long baselines } \\
\text { due to 20 m maximum delay }\end{array}$ \\
Calibration & $\begin{array}{l}\text { Typical uncertainties of } V^{2} \\
\text { in good observing conditions } \\
\text { Lower limit from maximum baseline length; } \\
\text { upper limit from bandwidth smearing }\end{array}$ \\
\hline
\end{tabular}

\section{THE CHARACTER OF MARK III DATA}

The fringe visibility amplitudes $V$ in observations of a binary with an interferometer are a result of the sky brightness distribution and the length and orientation of the baseline. In the sky plane, the brightness distribution consists of two small stellar disks with separation $\rho$ and position angle $\theta$. In the $u-v$ plane, where the coordinates are in wavelengths, the Fourier transform of the sky plane, the locus of the maxima of $V$ is a series of parallel straight lines (one passing through the origin) with separation proportional to $1 / \rho$ and position angle $\theta+\pi / 2$. The representation of the baseline in the $u-v$ plane is a vector at the origin with length proportional to $B / \lambda$ and position angle related to the baseline orientation and the hour angle.

As the Earth rotates, the baseline vector rotates in the $u-v$ plane, sweeping over the maxima and minima of $V$. The three observing wavelengths of the Mark III give rise to three effective baseline lengths, and thus three curves of visibility versus time, which contain the $(\rho, \theta)$ information. A sample curve from observations of $\alpha$ Equulei is shown in Figure 1.

The visibility data also contain, in principle, information about the diameters of the components and the magnitude differences at the observed wavelengths: the departure of the maximum $V$ from unity is related to the diameters of the components, and the ratio $V_{\min } / V_{\max }$ is related to the brightness ratio.

Using a Marquardt-Levenberg nonlinear least-squares algorithm, we fit models to the three sets of visibility data simultaneously to obtain estimates of $\rho$ and $\theta$ (under the assumption that changes in $\rho$ and $\theta$ during the night are nearly linear) and the brightness ratios at the observed wavelengths. For most 


\section{ALP EQU $800 . \mathrm{nm} \quad 900729$}

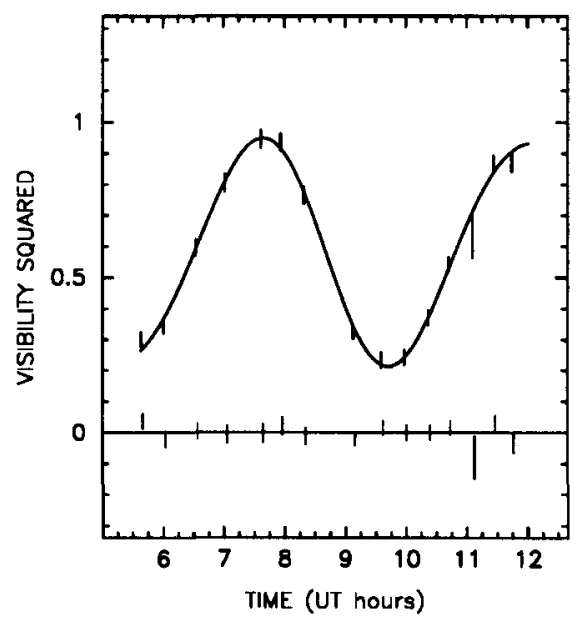

FIGURE 1. Squared visibility amplitudes $V^{2}$ for $\alpha$ Equ for 1990 July 29 are shown, with a curve of model visibilities for the best-fit $\rho, \theta$ for that date. Residuals are also shown. Bars are $\pm 1 \sigma$.

of the binaries observed with the Mark III, the departure of the maximum $V$ from unity is too small to yield reliable diameter estimates. Under the best conditions (good seeing, good S/N, and good hour angle coverage), the error ellipse for the separation can be as small as $0.2 \times 0.05$ mas, and the estimates of $\Delta m$ as good as 0.03 at $\lambda 800 \mathrm{~nm}$ or 0.06 at $\lambda 500 \mathrm{~nm}$. With the set of $(\rho, \theta)$ measurements, a visual orbit can be calculated by standard techniques. Uncertainties in the results from single-night data and from orbit fitting are estimated by mapping the hypersurface in parameter space on which the increase in $\chi^{2}$ from the minimum corresponds to the $68 \%$ confidence interval.

We are currently developing a more direct data-reduction technique in which the orbital elements and the brightness ratios are fitted simultaneously to all the visibilities from all of the nights of observation, and in which the uncertainties are estimated using a Monte Carlo technique.

\section{MASS MEASUREMENTS USING MARK III DATA}

Measurements of masses in four binary systems from Mark III data in conjunction with spectroscopic data are or will soon be in the literature. Preliminary results for four more systems are in varying degrees of preparation. These eight systems are listed in Table 2. Two of them are described in more detail below.

\section{1. $\alpha$ Equulei}

The first spectroscopic orbits for $\alpha$ Equulei (Deutsch 1954; Stickland 1976) indicated that the A dwarf in this system was more massive than the G giant, which was puzzling since the system is too wide for mass exchange to have taken place. However, re-examination of Stickland's data by Pike (1978) and further observations by Rosvick \& Scarfe (1991) have shown the reverse is true.

We observed $\alpha$ Equulei on 35 nights in 1989 and 1990, obtaining 29 nights of 
TABLE 2. Masses of Binaries Observed with the Mark III

\begin{tabular}{|c|c|c|c|}
\hline System & \multicolumn{2}{|c|}{ Masues $\left(\mathcal{M}_{\odot}\right)$} & Comment and Reference \\
\hline \multicolumn{4}{|c|}{ Final result: } \\
\hline$\beta$ Arietis & $2.34 \pm 0.10$ & $1.34 \pm 0.07$ & $\begin{array}{l}\text { Pan et al. }(1990) \text {; good agreement } \\
\text { with Sp of Tomkin \& Tran (1987) }\end{array}$ \\
\hline$\alpha$ Equulei & $2.13 \pm 0.29$ & $1.86 \pm 0.21$ & $\begin{array}{l}\text { Armstrong et al. (1992a); masses } \\
\text { and spectral types constrain age to } \\
\sim 0.5-0.8 \mathrm{Gyr}\end{array}$ \\
\hline$\phi$ Cygni & $2.521 \pm 0.085$ & $2.422 \pm 0.081$ & $\begin{array}{l}\text { Armstrong et al. (1992b); good } \\
\text { agreement with McAlister (1982) }\end{array}$ \\
\hline$\beta$ Trianguli & $3.90 \pm 0.15$ & $1.87 \pm 0.08$ & Vivekanand et al. (1992) \\
\hline \multicolumn{4}{|c|}{ Preliminary } \\
\hline $\begin{array}{l}\eta \text { Andromedae } \\
x \text { Andromedae } \\
\theta \text { Aquilae }\end{array}$ & $\begin{array}{l}2.65 \pm 0.1 \\
30.8 \pm 0.7 \\
0.3 \text { or } 0.8\end{array}$ & $\begin{array}{l}2.40 \pm 0.1 \\
12.5 \pm 0.3 \\
0.2 \text { or } 0.6\end{array}$ & $\begin{array}{l}\text { Spectroscopic and Mark III values of } \\
e \text { disagree }\end{array}$ \\
\hline$\eta$ Pegasi & 3 & 3 & \\
\hline
\end{tabular}

useful data. Separations ranged from 9.6 to 12.4 mas, with measured magnitude differences ranging from $1.2 \mathrm{mag}$ at $\lambda 800 \mathrm{~nm}$ to $\approx 0.3 \mathrm{mag}$ at $\lambda 500 \mathrm{~nm}$. The adopted angular diameters (after the procedure of Mozurkewich et al. 1991) of 0.34 and 1.5 mas at $\lambda 800 \mathrm{~nm}$ were too small to fit with Mark III data. The orbit is shown in Figure 2.

The masses and distance determined from combining our data with those of Rosvick \& Scarfe, and the magnitude differences from our data, permit us to place both stars on the color-magnitude diagram and compare their positions with predictions of stellar evolution models. We used the models of VandenBerg (1985), and determined that the masses were consistent with a single age for the system of $\approx 0.5$ to $0.8 \mathrm{Gyr}$. Results for $\alpha$ Equulei are presented in Table 3.

\section{2. $\phi$ Cygni}

$\phi$ Cygni is a system with two G-type giants; consequently the radial velocity data are quite good. Rach \& Herbig (1961) give $K_{1,2}=26.79$ and 27.88 $\mathrm{km} \mathrm{s}^{-1}$ with $0.05 \mathrm{~km} \mathrm{~s}^{-1}$ probable error. McAlister (1982) observed this system with speckle interferometry, obtaining six measurements and four upper limits on the separation. Using four orbital elements from the speckle results with the radial velocity results of Rach \& Herbig (1961), he obtained masses of $2.50 \pm$ $0.09 \mathcal{M}_{\odot}$ and $2.39 \pm 0.08 \mathcal{M}_{\odot}$.

We observed $\phi$ Cygni on 74 occasions from 1989 to 1991, obtaining 41 nights with enough data for determining separations. The separations ranged from 4.7 to 31 mas; the value of the semi-major axis of the error ellipse for the best 16 nights was less than 0.5 mas. Magnitude differences were zero within $0.03 \mathrm{mag}$ $($ at $\lambda 800 \mathrm{~nm})$ to $0.1 \mathrm{mag}($ at $\lambda 500 \mathrm{~nm})$. Angular diameters were too small to determine from the data, and were taken to be 0.87 mas at $\lambda 800 \mathrm{~nm}$ after the procedure given by Mozurkewich et al. (1991). 


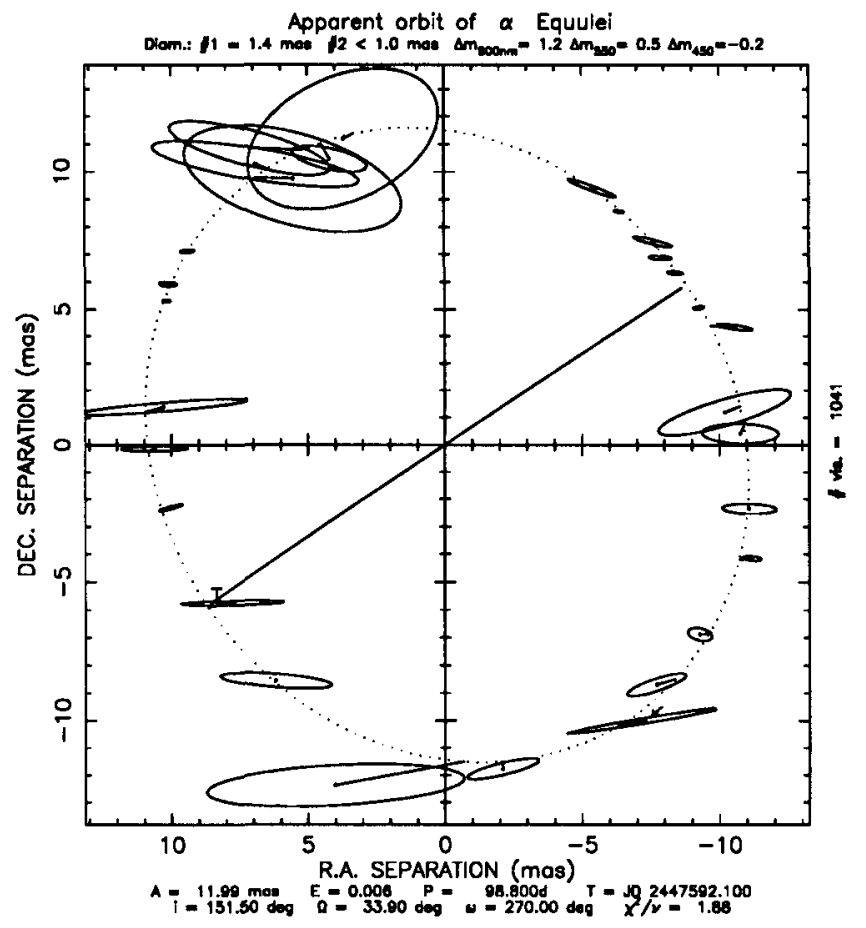

FIGURE 2. The best-fit orbit for $\alpha$ Equ as projected on the sky, with $1 \sigma$ error ellipses for individual nights. Lines connect the observed points with their model positions on the best-fit orbit. Small dots show the orbit at intervals of $P / 200$.

TABLE 3. Orbital Elements and System Parameters for $\alpha$ Equulei

\begin{tabular}{|ll|ll|}
\hline \multicolumn{2}{c|}{ Orbital Elements } & \multicolumn{2}{c|}{ System Parameters } \\
\hline$a$ & $11.987 \pm 0.078 \mathrm{mas}$ & $a_{A}+a_{G}$ & $0.663 \pm 0.027 \mathrm{AU}$ \\
$e$ & $0.0056 \pm 0.0034$ & $\mathcal{M}_{A}$ & $1.86 \pm 0.21 \mathcal{M}_{\odot}$ \\
$P$ & $98.800 \pm 0.004^{a}$ & $\mathcal{M}_{G}$ & $2.13 \pm 0.29 \mathcal{M}_{\odot}$ \\
$T$ & $\mathrm{JD} 2447592.1 \pm 3.6$ & $D$ & $55.3 \pm 2.3 \mathrm{pc}$ \\
$i$ & $151^{\circ} .5 \pm 10$. & $M_{V, A}$ & $1.21 \pm 0.09$ \\
$\Omega$ & $33^{\circ} .9 \pm 2^{\circ} .2$ & $M_{V, G}$ & $0.75 \pm 0.10$ \\
$\omega$ & $270^{\circ} \pm 14^{\circ}$ & & \\
\hline \multicolumn{3}{|c}{ "From Rosvick \& Scarfe (1991) } \\
\hline
\end{tabular}



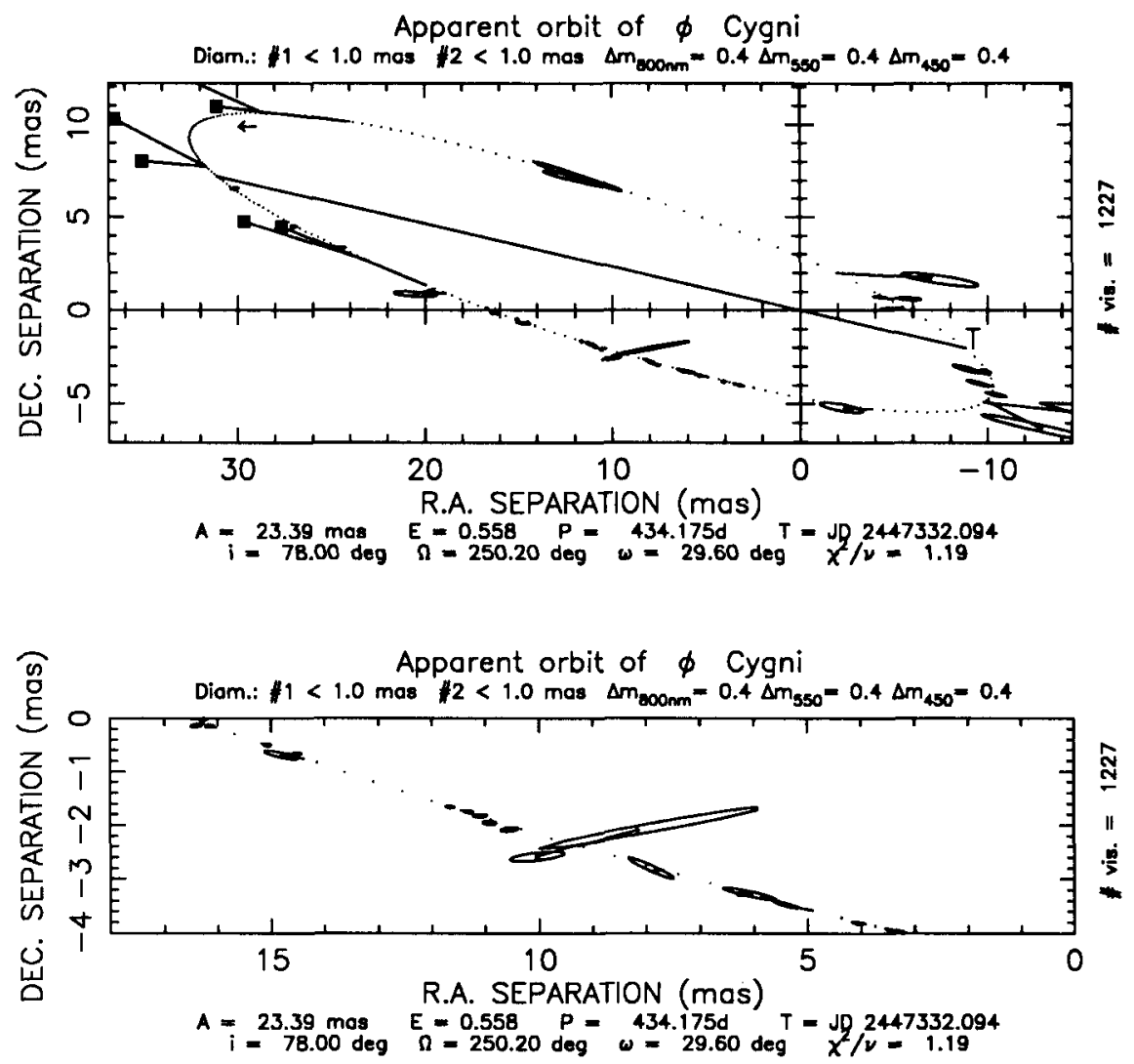

FIGURE 3. The upper panel shows best-fit orbit for $\phi$ Cyg as with Figure 2. Five speckle points observed by McAlister (1982) are shown as squares. The lower panel shows a detail of part of the orbit.

We used the Mark III data to solve for six of the seven orbital elements, adopting the period given by Rach \& Herbig (1961); in addition, we redetermined the spectroscopic elements from the data of Rach \& Herbig. The orbit is shown in Figure 3; the orbital elements from the Mark III data and the system parameters deduced from the combination of Mark III and spectroscopic data are shown in Table 4.

\section{CONCLUSION}

Results from the Mark III interferometer, which was originally intended for astrometry, are starting to contribute to the list of known stellar masses. The uncertainties for two systems are less than 5\%, and thus begin to approach the level of precision cited by Andersen (1991) as necessary for detailed tests of stellar evolution models and metallicity determinations. Future results may 
TABLE 4. Orbital Elements and System Parameters for $\phi$ Cygni

\begin{tabular}{|ll|ll|}
\hline \multicolumn{2}{|c|}{ Orbital Elements } & \multicolumn{2}{|c|}{ System Parameters } \\
\hline$a$ & $23.39 \pm 0.45 \mathrm{mas}$ & $a_{1}+a_{2}$ & $1.912 \pm 0.020 \mathrm{AU}$ \\
$e$ & $0.558 \pm 0.010$ & $\mathcal{M}_{1}$ & $2.545 \pm 0.085 \mathcal{M}_{\odot}$ \\
$P$ & $434.175 \pm 0.032^{a}$ & $\mathcal{M}_{2}$ & $2.445 \pm 0.081 \mathcal{M}_{\odot}$ \\
$T$ & $\mathrm{JD} 2447332.09 \pm 0.63$ & $D$ & $81.8 \pm 1.0 \mathrm{pc}$ \\
$\boldsymbol{i}$ & $78^{\circ} .0 \pm 0^{\circ} .36$ & $M_{V, 1}$ & $0.92 \pm 0.1$ \\
$\Omega$ & $250^{\circ} .2 \pm 00.25$ & $M_{V, 2}$ & $1.17 \pm 0.1$ \\
$\omega$ & $29^{\circ} .6 \pm 1^{\circ} .7$ & \\
\hline \multicolumn{2}{|c}{ 'From Rach \& Herbig (1961) } \\
\hline
\end{tabular}

amount to mass determinations in $\approx 10$ to 20 systems before the Mark III is superseded by more advanced interferometers. One such instrument is being planned by NRL: a six-element $Y$-shaped array with $35 \mathrm{~cm}$ apertures and a maximum baseline length of $430 \mathrm{~m}$. This interferometer will be co-located with the USNO astrometric interferometer; groundbreaking for both should take place during the summer of 1992 .

\section{REFERENCES}

Andersen, J. 1991, $A R A \& A, 3,91$

Anderson, J.A. 1920, $A p J, 51,263$

Armstrong, J.T., Mozurkewich, D., Vivekanand, M., Simon, R.S., Denison, C.S., Johnston, K.J., Pan X.-P., Shao, M., \& Colavita, M.M. 1992a, AJ, 104, 241

Armstrong, J.T., Mosurkewich, D., Vivekanand, M., Simon, R.S., Denison, C.S., Johnston, K.J., Pan X.-P., Shao, M., \& Colavita, M.M. 1992b, in preparation

Deutsch, A.J. 1954, PASP, 66, 58

McAlister, H.A. 1982, $A J, 87,563$

Mosurkewich, D., Johnston, K.J., Simon, R.S., Bowers, P.F., Gaume, R A., Hutter, D.J., Colavita, M.M., \& Shao, M. 1991, $A J, 101,2207$

Pan X.-P., Shao, M., Colavita, M.M., Mozurkewich, D., Simon, R.S., \& Johnston, K.J. 1990, ApJ, 356, 641

Pike, C.D. 1878, MNRAS, 184, 265

Rach, R.A. \& Herbig, G. H. 1961, ApJ, 133, 143

Rosvick, J.M. \& Scarfe, C.D. 1991, MNRAS, 252, 68

Shao, M., Colavita, M.M., Hines, B.E., Staelin, D.H., Hutter, D.J., Johnston, K.J., Mosurkewich, D., Simon, R.S., Hershey, J.L., Hughes, J.A., \& Kaplan, G.H. 1988, $A \& A, 193,357$

Stickland, D.J. 1976, MNRAS, 175, 473

Tomkin, J. \& Tran, H. 1987, $A J, 94,1664$

VandenBerg, D.A. 1985, ApJS, 58, 711

Vivekanand, M., Armstrong, J.T., Pan X.-P., Shao, M., Mozurkewich, D., Colavita, M.M., Denison, C. S., Simon, R. S., \& Johnston, K. J. 1992 (in preparation) 


\section{DISCUSSION}

TOKOVININ: Do you consider the operation of your future interferometers in the near IR at $2.2 \mu \mathrm{m}$ for example?

ARMSTRONG: Operations at $2.2 \mu \mathrm{m}$ have been considered. Our first "upgrades" will be to build the full instruments that we have designed, with separate astrometric and imaging instruments, with $250 \mathrm{~m}$ arms for the $\mathrm{Y}$ of the imaging instrument, with beam compressors so we can use $35 \mathrm{~cm}$ apertures. The $2.2 \mu \mathrm{m}$ capability will be high on the list when the full system is complete.

DAVIS: Your maximum visibilities appear to be normalized to unity. What are the typical raw visibilities that you measure for unresolved sources?

ARMSTRONG: The visibilities shown have been normalized by observations of unresolved single stars. Typical raw visibilities are (for reasonably good seeing: Mt. Wilson in late summer and early fall $) \mathrm{V}(800 \mathrm{~nm})=0.8$ and $\mathrm{V}(500 \mathrm{~nm})=0.6$. For the very best nights, these increase to 0.9 and 0.7 .

SCARFE: Do you observe resolvable single stars, and what is your time resolution? It could be that comparison with the work of the precise radial velocity groups might lead to some interesting astrophysics.

ARMSTRONG: I should have mentioned the three observatoins programs at the Mark III: astrometry (for which the instrument was originally conceived), the binary research I've described, and stellar diameter measurements. Dave Mozurkewich of our grouo at NRL is reducing data for about 70 stellar diameters. Andreas Quirrenbach has done some observations of $\mathrm{M}$ giants in the TiO bands and in the continuum, and we also have some observations of Mira, which show it to be asymmetric, and of Nova Cygni 1992 (but only one night). The time scale for diameter measurements is the time for changing baselines; we can change between a variable-baseline pair $(3$ to $31 \mathrm{~m})$ to an astrometric baseline $(12 \mathrm{~m})$ in about 15 minutes. But, I would have to think about whether our precision in the diameter measurements would be sufficient to make an interesting companion to the precise radial velocity measurements.

GHEZ: You mentioned that you have seen Mira to be asymmetric. Do these measurements agree with Margarita Karovska's results? This would be a good confirmation of speckle imaging techniques.

QUIRRENBACH: Our data (in press in A\&A) show an asymmetry with an axial ratio of about 0.8 , in agreement with $M$. Karovska's results. We also find variations of the position angle of about $50^{\circ}$ within only two months. This also agrees qualitatively with her findings; a more detailed comparison is not possible since her measurements were more widely spaced and not simultaneous with ours.

POVEDA: Your results are very impressive! Why are you not considering scaling up the aperture of the primary mirrors to reach substantially fainter stars?

ARMSTRONG: There are two reasons: 1) The atmospheric coherence length, $r_{0}$, is generally of the order of 10 to $40 \mathrm{~cm}$. For a fringe tracking system, we limit ourselves to $r_{0}$ apertures in order to have a single set of fringes 
to track. Having larger apertures does not extend the magnitude limit because the fringes from different speckle will reduce fringe contrast as well as making calibration difficult. 2) One can make single-ro instruments by applying higherorder adaptive-optics corrections to large apertures, perhaps using laser guide stars (the Mark III, our future instruments, and other interferometers already correct for wavefront tilt), but there it's a question of money.

McALISTER: Speckle has gained some degree of respectability by measuring many visual binaries to overlap with well established methods. Other than $\phi$ Cygni, have you obtained observations of speckle binaries in order to provide similar overlap?

ARMSTRONG: Our initial work has been primarily on binaries closer than those resolvable by speckle interferometry on current telescopes. We have been reminded-by Geoff Douglass among others-of the danger of overlooking binaries that can be observed with speckle techniques, and we have started to observe several of these stars. We welcome suggestions of appropriate stars to add to our observing list. In practical terms, it is better for us to measure stars separated by less than $\sim 200$ mas; wider separations are difficult because of the bandwidth smearing and the rate of binary-fringe visibility variation. The stars that appear in your catalog of interferometric measurements in the table under stars first resolved with interferometry for which we have a significant amount of data are: 12 Per, $\gamma$ Per, $\beta$ Per, $\eta$ Vir, $\beta$ CrB, $\chi$ Dra, $\phi$ Cyg, $\delta$ Sge, $\beta$ Cep, $\xi$ Cep, and $\alpha$ Aur. Stars with a significant amount of Mark III data but with few speckle separations are: $\xi^{1}$ Cet, 41 Ari, $\tau$ Per, $\gamma$ Tau, o Leo, $\beta$ Her, $\eta$ Peg. 